\title{
Using rheometry to determine nucleation density in a colored system containing a nucleating agent
}

\author{
Zhe Ma $\cdot$ Rudi J. A. Steenbakkers • \\ Julien Giboz • Gerrit W. M. Peters
}

Received: 11 June 2010 / Revised: 24 September 2010 / Accepted: 11 November 2010 / Published online: 8 December 2010

(C) The Author(s) 2010. This article is published with open access at Springerlink.com

\begin{abstract}
A new suspension-based rheological method was applied to experimentally study the crystallization of a nucleating agent (NA) filled isotactic polypropylene. This method allows for determination of point nucleation densities where other methods fail. For example, optical microscopy can fail because nucleation densities become too high to be counted (materials with effective NA) or crystallites are not easily visible (colored materials), while differential scanning calorimetry does not allow the effect of flow to be studied. Both quiescent and mild-shear-induced crystallization were investigated. The results show that the addition of a nucleating agent increases the nucleation density by six decades for quiescent crystallization. The effect of shear on crystallization in the presence of a nucleating agent was assessed, and it is demonstrated that, at least for this system, the effect of shear is much smaller than the effect of the nucleating agent.
\end{abstract}

Keywords Polymer crystallization - Nucleation • Nucleating agent $\cdot$ Shear $\cdot$ Flow-induced crystallization $\cdot$ Rheometry

This paper was presented at the 6th Annual European Rheology Conference, April 7-9 2010, Göteborg, Sweden.

Z. Ma · R. J. A. Steenbakkers · G. W. M. Peters $(\bowtie)$ Materials Technology, Department of Mechanical Engineering, Eindhoven University of Technology, P.O. Box 513, 5600MB Eindhoven, The Netherlands e-mail: g.w.m.peters@tue.nl

J. Giboz

LMOPS, CNRS UMR5041, Université de Savoie,

73376 Le Bourget-du-Lac, France

\section{Introduction}

Semi-crystalline polymers cover over two thirds of the products used in our daily life. Adding a nucleating agent to a semi-crystalline polymer is a common way to control crystallization and tailor mechanical and optical properties (Kristiansen et al. 2003, 2005; Kurja and Mehl 2001; Menyhárd et al. 2009). The interface between the nucleating agent and the polymer melt has a high surface energy, which reduces the energy barrier associated with the formation of a nucleus. Introducing a large amount of nucleating agent particles therefore greatly increases the nucleation density and consequently the rate of crystallization (Wunderlich 1973). In general, a higher nucleation density leads to more desirable properties. Several methods are available to determine the nucleation density in polymer crystallization. These include (a) microscopy to directly count the number of nuclei in the melt, (b) differential scanning calorimetry (DSC; Lamberti 2004), or (c) dilatometry (Binsbergen and De Lange 1970). The latter two indirectly measure the crystallization evolution in order to calculate the nucleation density using a kinetics equation. However, for colored nucleating agent systems, the nucleation density can become too large to be counted and also can change the optical properties dramatically (Menyhárd et al. 2009), making quantification of the nucleation density difficult with optical microscopy (Fig. 1).

Flow is another crucial factor affecting polymer crystallization, especially the nucleation step. The number density of nuclei can increase dramatically (Housmans et al. 2009; Zuidema et al. 2001), and for a strong enough flow, the formation of shish-kebab structures will occur (Zhu and Edward 2004). The combined 


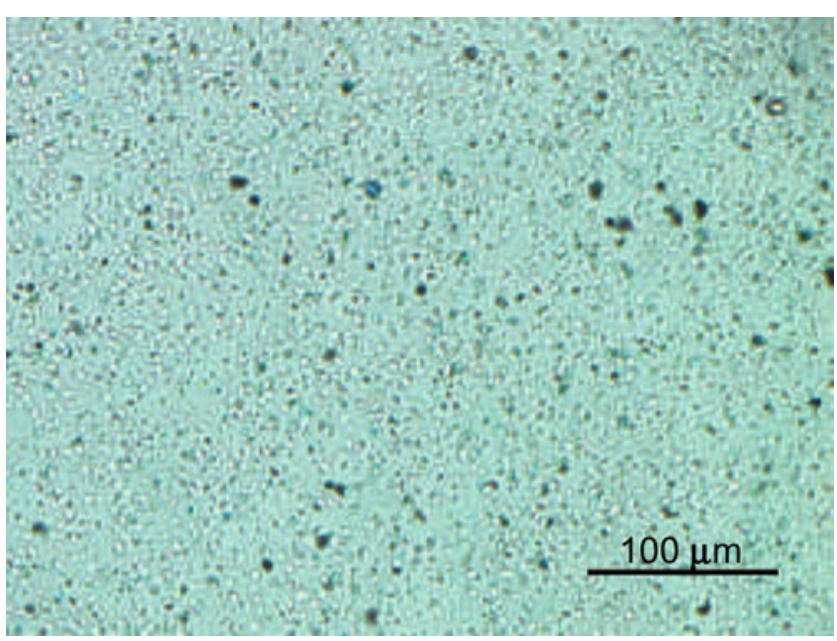

Fig. 1 Morphology during quiescent crystallization at $T=151^{\circ} \mathrm{C}$

effect of nucleating agent and flow has hardly been studied. In this work, we will restrict ourselves to pointlike nucleation which will occur for moderate flow only. How much a moderate flow will change the kinetics of nucleating agent crystallization depends on the type of nucleating agent (Byelov et al. 2008). Knowledge of the individual effects of nucleating agent and shear on nucleation density is a prerequisite for understanding the combined nucleation mechanism and to provide input for models to predict resulting structures.

To achieve this goal, conventional DSC and classical dilatometry are not suited since they do not allow one to impose a flow on the material. A new type of dilatometer, the Pirouette (http://www.imetechnologies.nl), developed in our group (Forstner et al. 2009; Van der Beek et al. 2006), does allow for applying shear to the sample and thus for using the measured timedependent specific volume for our purpose: determining the nucleation density. However, in this paper, we will use a rheometer that first serves as flow device (with the possibility to vary the shear rate and the shear time) and, subsequently, as a mechanical spectrometer that measures the changing complex modulus due to the progressing crystallization process. Using an analytical relation between space filling and the modulus, it is possible to determine the nucleation density. This solves both problems: dealing with large numbers of nuclei and poor visibility of crystalline structures. The aim of this study is to explain this approach and to demonstrate its effectiveness for colored nucleating agent systems, for both quiescent and flow-induced crystallization.

\section{Experimental methods}

The polymer used in this study is an isotactic polypropylene (iPP; HD601CF, Borealis, previously known as HD120MO). It has a weight average molecular weight $M_{\mathrm{w}}=365 \mathrm{~kg} / \mathrm{mol}$ and a polydispersity $M_{\mathrm{w}} / M_{\mathrm{n}}=5.4$ (Housmans et al. 2009). The nominal melting temperature is $163^{\circ} \mathrm{C}$. The polymer was compounded with an organic nucleating agent, U-Phthalocyanine of molecular weight $310 \mathrm{~kg} / \mathrm{mol}$ (C. Hadinata 2009, personal communication) at a concentration of $0.2 \mathrm{wt} \%$. This nucleating agent was also used by Lee Wo and Tanner (2010) who found non-spherical crystallites that we did not observe, see "Results and discussion". From the neat iPP and the artificially nucleated material (NA-iPP), 1.1-mm-thick plates were injectionmolded and from those plates circular disks were cut with a diameter of $8 \mathrm{~mm}$. The NA-iPP samples were blue due to the coloring effect of the nucleating agent.

For the rheological measurements, a Rheometrics ARES rheometer with a plate-plate geometry was used. Samples were first heated to $230^{\circ} \mathrm{C}$ and kept on that temperature for $10 \mathrm{~min}$ to erase the thermal and mechanical history. Next, the melt was cooled to the desired temperature at a rate of $15^{\circ} \mathrm{C} / \mathrm{min}$ and kept at this temperature for the isothermal crystallization. During cooling, gap adjustment was performed continuously to compensate for the thermal shrinkage of tools and sample. Two minutes of delay time was used to equilibrate the sample temperature before starting the dynamic measurements. Temperatures for crystallization were chosen between $133^{\circ} \mathrm{C}$ and $140^{\circ} \mathrm{C}$ for the neat iPP and between $143^{\circ} \mathrm{C}$ and $151^{\circ} \mathrm{C}$ for the NA-iPP. Experiments with shear were carried out at temperatures of $148^{\circ} \mathrm{C}$ and $151^{\circ} \mathrm{C}$. Steady shear at a rate of $\dot{\gamma}=60 \mathrm{~s}^{-1}$ was imposed for shear times $t_{\mathrm{s}}=2,4$, and $6 \mathrm{~s}$. Small-amplitude oscillatory shear measurements were employed to track the time evolution of the storage modulus $\left(G^{\prime}\right)$ and loss modulus $\left(G^{\prime \prime}\right)$ using an angular frequency of $5 \mathrm{rad} / \mathrm{s}$ and a strain of $0.5 \%$. All experiments were performed in an $\mathrm{N}_{2}$ atmosphere to prevent the material from degrading.

Polypropylene can crystallize in different phases. To check if both the neat iPP and the NA-iPP had crystallized in the $\alpha$-phase, the samples were analyzed afterwards using X-ray scattering. Wide-angle X-ray diffraction (WAXD) measurements were carried out at the Dutch-Belgian (DUBBLE) beamline BM26 of the European Synchrotron Radiation Facility in Grenoble, France. A Photonica CCD detector with 2,004 $\times$ 1,335 pixels of $44 \mu \mathrm{m} \times 44 \mu \mathrm{m}$ was placed at $178 \mathrm{~mm}$. The wavelength was $1.033 \AA$ and the exposure time was 
10 s. WAXD data were integrated with the software FIT2D.

\section{Data analysis}

A linear viscoelastic version of the three-dimensional generalized self-consistent method of Christensen and Lo (1979) is used to couple the amount of space filling, caused by point-like nucleation and spherulitic growth, to the measured dynamic (or complex) modulus. See also Christensen and Lo (1986) and Christensen (1990). This model has been validated experimentally in our previous work (Steenbakkers and Peters 2008). If spherulites are formed, the relative dynamic modulus $f_{G}^{*}=G^{*} / G_{0}^{*}$ can be obtained from

$A^{*} f_{G}^{* 2}+B^{*} f_{G}^{*}+C^{*}=0$,

where $G^{*}$ and $G_{0}^{*}$ are the complex dynamic modulus of the suspension and the amorphous phase, respectively. The complex coefficients $A^{*}, B^{*}$, and $C^{*}$ depend on space filling $\phi$, the ratio of the complex moduli of the amorphous $\left(G_{0}^{*}\right)$ and semi-crystalline phase $\left(G_{1}^{*}\right)$, and the Poisson ratios of both phases, $v_{0}$ and $v_{1}$. Notice that all moduli are frequency and temperature dependent. Expressions for the coefficients are given in Appendix A of Steenbakkers and Peters (2008). In this case, space filling is the unknown and is obtained by minimizing Eq. 1 using the measured $f_{G}^{*}$.

Next the space filling has to be related to the nucleation density. For a fixed number density of nuclei $N(T)$, the Kolmogorov-Avrami-Evans equation (Avrami 1939, 1940; Evans 1945; Kolmogorov 1937) describes the progress of space filling in time during isothermal crystallization at temperature $T$,

$\phi(t, T)=1-\exp \left(-\frac{4 \pi}{3} N(T) G^{3}(T) t^{3}\right)$

from which the nucleation density can be obtained:

$N(T)=-\frac{3 \ln (1-\phi)}{4 \pi G^{3}(T) t^{3}}$

with growth rate $G(T)$ depending on the temperature only. The main requirement here is that the growth rate is independent of the nucleation density, even when this density is influenced by a nucleating agent or by flow. Flow experiments are so-called short-term shear experiments; the flow can generate extra nuclei but the growth takes place after the flow has stopped. The growth rate for iPP is well-known (Eder and JaneschitzKriegl 1997). Note that there are different empirical relations describing the temperature dependence of the growth rate, see Eq. 4 (Zuidema et al. 2001) and Eq. 5 (Hoffman et al. 1976):

$$
\begin{aligned}
G(T)= & G_{\mathrm{ref}} \exp \left[-c_{G}\left(T-T_{G, \mathrm{ref}}\right)^{2}\right] \\
G(T)= & G_{0} \exp \left[-\frac{U^{*}}{R\left(T-T_{\mathrm{g}}+T_{\infty}\right)}\right] \\
& \times \exp \left[-\frac{\kappa_{G} T_{\mathrm{m}}^{2}\left(T_{\mathrm{m}}+T\right)}{2 T^{2}\left(T_{\mathrm{m}}-T\right)}\right]
\end{aligned}
$$

One could think of using other approaches by applying more simple models (Boutahar et al. 1996, 1998; Tanner 2002, 2003; van Ruth et al. 2006) or empirically based scaling laws between the space filling and the storage modulus (see Coppola et al. 2006; Khanna 1993; Pogodina et al. 1999). However, it was demonstrated in Steenbakkers and Peters (2008) that such approaches do perform less than the suspension model as used here, and therefore, we will not apply them to our results. Summarizing, by measuring the complex modulus $G^{*}\left(t, T, \dot{\gamma}, t_{\mathrm{s}}\right)$ for different temperatures, shear rates $\dot{\gamma}$, and shear times $t_{\mathrm{s}}$, we can determine the space filling $\phi(t, T)$ using Eq. 1 and the nucleation density $N\left(T, \dot{\gamma}, t_{\mathrm{s}}\right)$ using Eq. 3, provided that only spherulitic growth from predetermined point-like nuclei occurs.

\section{Results and discussion}

Determination of the nucleation density for NA-iPP

First of all, Fig. 1 shows that the structures formed during crystallization with nucleating agent do not show any unusual structure as was found by Lee Wo and Tanner (2010) for a similar system (a commercial iPP with different concentrations of U-Phthalocyanine), so the above method can be applied to convert the time evolution of the rheological properties into kinetics of space filling. Figure 2 shows the time evolution of the storage modulus $G^{\prime}$ of the nucleating agent system during crystallization at different temperatures. First of all, it should be noticed that for $T=145^{\circ} \mathrm{C}$ the initial value of $G^{\prime}$ is not the same as those for the other temperatures. The method is based on the assumption of isothermal crystallization. If the degree of undercooling is large with respect to the cooling rate, crystallization already sets in during cooling. The problem is made 


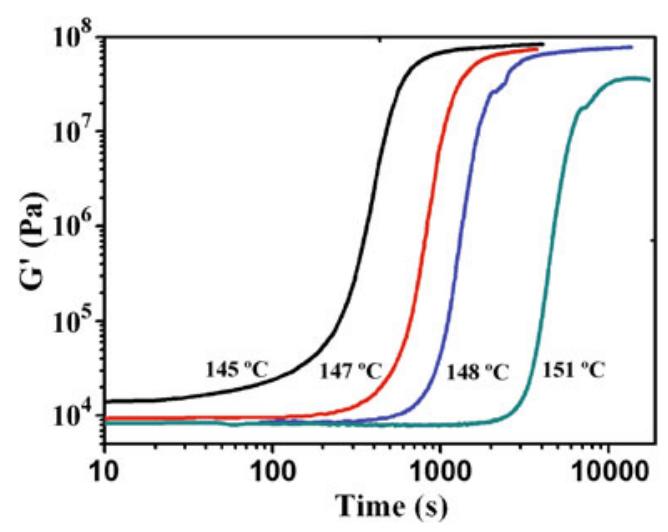

Fig. 2 Time evolution of the storage modulus for NA-iPP samples crystallized at different temperatures

even worse due to the 2-min delay time we used in order to equilibrate the sample temperature. This is the case for $T=145^{\circ} \mathrm{C}$; crystallization has already started before we begin to track the rheological evolution (indicated by an increased initial value of $G^{\prime}$ ). Faster cooling rates are required when studying higher levels of undercooling. Nevertheless, we still want to show this result to demonstrate the limitations of the method.

The related space filling, determined by Eq. 1, is shown in Fig. 3. For the high experimental temperature range used here, the growth rate is better captured by Eq. 4 (Eder and Janeschitz-Kriegl 1997; Zuidema et al. 2001), so this expression was applied. Figure 4 shows the diffraction patterns of NA-iPP samples after quiescent crystallization and demonstrates that nucleating agent crystallization results in the same $\alpha$ modification as crystallization of the neat iPP. Consequently, the same growth kinetics, Eq. 4, applies. The number density of nuclei determined by Eq. 3 is plotted versus space filling in Fig. 5. It is nearly constant between $\phi \approx 0.1$ and $\phi \approx 0.9$, except for the experiment at $T=$

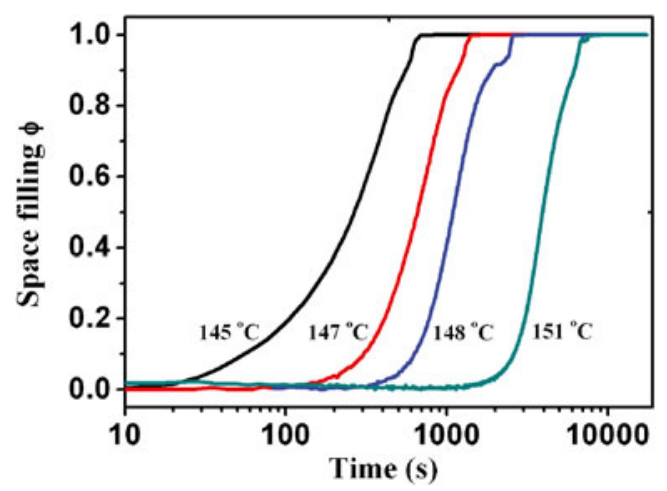

Fig. 3 Time evolution of space filling derived from the dynamic modulus for NA-iPP samples crystallized at different temperatures

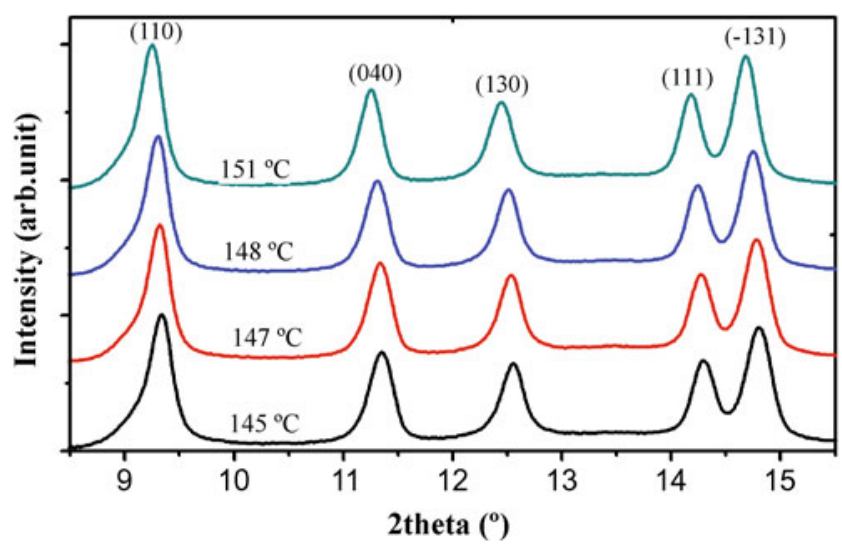

Fig. 4 One-dimensional WAXD curves of NA-iPP samples crystallized at different temperatures

$145^{\circ} \mathrm{C}$, where crystallization has already set in before the dynamic measurements have started. Much higher values are found in the early stage, but $N(T)$ becomes nearly constant when $\phi \approx 0.4$ for all temperatures. The influence of the unknown initial space filling is relatively smaller for higher degrees of space filling, i.e., in later stages of the process. We have taken the average value for a space filling between $\phi=0.5$ and $\phi=0.9$ as a reasonable approximation. Nucleation densities from all experiments are plotted as a function of the experimental temperature in Fig. 6. It can be concluded that this type of nucleating agent is very effective as it increases the nucleation density by up to six decades. Notice that the temperature dependency of the nucleation density is very similar for the neat iPP and the NA-iPP and that the results for $T=145^{\circ} \mathrm{C}$, although less reliable for $\phi<0.4$, are well in line with the results for higher temperatures. Results for relatively low temperatures should be treated with some caution.

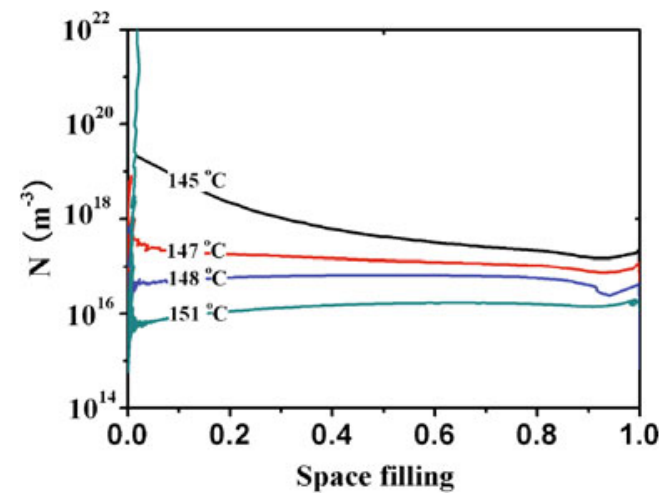

Fig. 5 Nucleation density versus space filling for NA-iPP samples crystallized at different temperatures 


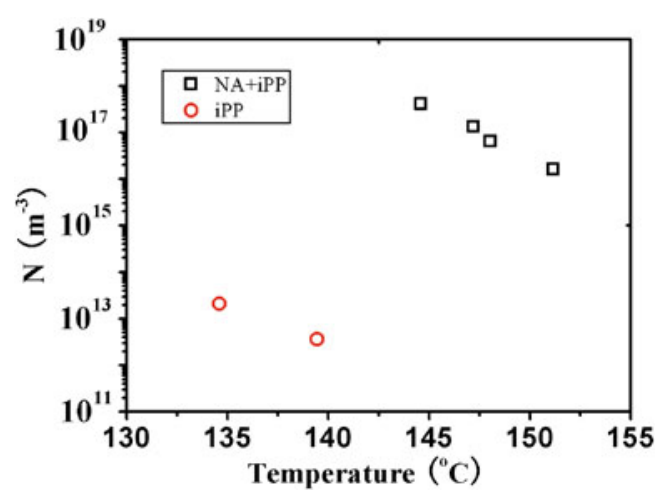

Fig. 6 Nucleation density of iPP and NA-iPP versus temperature

\section{Reproducibility}

First we compare the results of Housmans et al. (2009), obtained with the same method and for the same iPP, to the results presented here. For a temperature $T=138^{\circ} \mathrm{C}$, they found, for quiescent conditions, a nucleation density $N=8 \times 10^{11} \mathrm{~m}^{-3}$, while we obtain the (interpolated) value $N=6 \times 10^{12} \mathrm{~m}^{-3}$. We ascribe this difference to the sample preparation procedure. Our neat iPP and nucleated samples were processed by injection molding, which means that the sample preparation step already induced extra nuclei due to the applied (uncontrolled) flow. The samples used by Housmans et al. (2009) were prepared by means of compression molding.

We repeated our experiments to check for reproducibility. The time lapse between the two series of experiments was about 8 weeks. The results of these repeated experiments (second series) are shown in Fig. 7 , together with the previous results (first series). We want to stress the importance of a good temperature control. An error of $1{ }^{\circ} \mathrm{C}$ typically gives a factor two difference in the number density of nuclei. Notice that

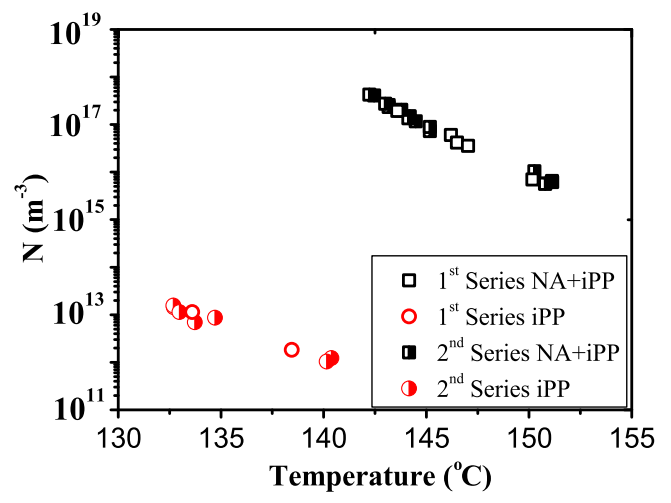

Fig. 7 Nucleation density of iPP and NA-iPP versus temperature for two series of experiments in the second series of experiments we managed to get good results for temperatures as low as $T=143^{\circ} \mathrm{C}$ while in the first series we already encountered problems at $T=145^{\circ} \mathrm{C}$.

\section{Effect of mild flow}

Figure 8 shows the evolution of $G^{\prime}$ during crystallization at $148^{\circ} \mathrm{C}$ under quiescent conditions and after shear (fixed shear rate and variable shear time). For a shear rate of $60 \mathrm{~s}^{-1}$, a shear time of $2 \mathrm{~s}$ shows clearly accelerated kinetics. Further increase of the shear time to $4 \mathrm{~s}$ does not change the kinetics much. The acceleration of crystallization seems to become (nearly) independent of the shear time beyond $4 \mathrm{~s}$, indicating that the shear-enhanced point-like nucleation saturates. This effect was also observed by Housmans et al. (2009) for this and two other iPPs. The time evolution of the storage modulus of the sheared nucleating agent system has the same shape as in the quiescent nucleating agent system, meaning that the growth mechanism is the same and only spherulites are formed. This implies that our method can still be applied to determine space filling for crystallization after shear. Moreover, the results can be compared to those of Housmans et al. (2009).

For flow-induced crystallization experiments, an adequate measure of the flow strength is the Weissenberg number, based on the stretch relaxation time of the HMW tail of the MWD distribution:

$W i_{\mathrm{s}}(T, \dot{\gamma})=\tau_{\mathrm{s}}^{\mathrm{HMW}}(T) \dot{\gamma}$

Recent modeling work (Steenbakkers 2009) suggests that the creation rate of point-like nucleation precursors depends not only on the average stretch of the HMW molecules but also explicitly on the temperature: The prefactor of the creation rate was found to be

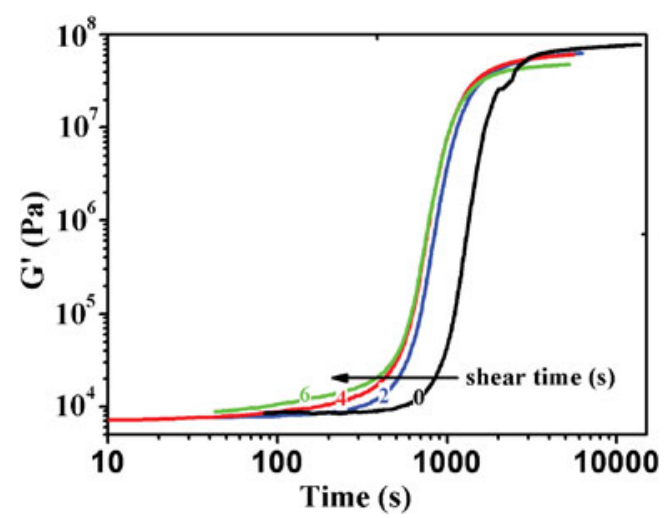

Fig. 8 Time evolution of the storage modulus for NA-iPP under quiescent conditions and after short term shear $\left(\dot{\gamma}=60 \mathrm{~s}^{-1}, t_{\mathrm{s}}=\right.$ $2,4,6 \mathrm{~s}$ ) at $T=148^{\circ} \mathrm{C}$ 


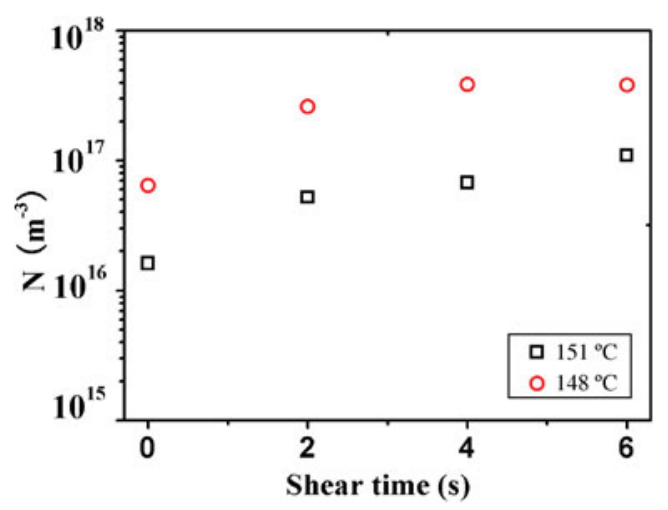

Fig. 9 Nucleation density of NA-iPP under quiescent conditions and after shear at $T=148{ }^{\circ} \mathrm{C}$ and $T=151^{\circ} \mathrm{C}\left(\dot{\gamma}=60 \mathrm{~s}^{-1}\right)$

proportional to the time-temperature shift factor $a_{T}$. Therefore, we use $a_{T} W i_{\mathrm{s}}$ as a criterion to compare experiments, which scales as $a_{T}^{2}$. From the linear viscoelastic data and the stretch relaxation time $\tau_{\mathrm{s}}^{\mathrm{HMW}}$ reported by Housmans et al. (2009), we find $W i_{\mathrm{s}}\left(148^{\circ} \mathrm{C}\right)=$ $13, W i_{\mathrm{s}}\left(151^{\circ} \mathrm{C}\right)=12$, and

$\frac{a_{T}\left(151^{\circ} \mathrm{C}\right) W i_{\mathrm{s}}\left(151^{\circ} \mathrm{C}\right)}{a_{T}\left(138^{\circ} \mathrm{C}\right) W i_{\mathrm{s}}\left(138^{\circ} \mathrm{C}\right)}=1.1$,

where $W i_{\mathrm{s}}\left(138^{\circ} \mathrm{C}\right)=8$ for the strongest flow applied by Housmans et al. (2009) to the same neat iPP as used here. Hence, these two data sets are reasonably comparable in terms of flow conditions.

The calculated nucleation densities are plotted in Fig. 9. The saturated nucleation density after shear is around $40 \times 10^{16} \mathrm{~m}^{-3}$, six times higher than $7 \times$ $10^{16} \mathrm{~m}^{-3}$ for quiescent crystallization, i.e., adding typically $\sim 10^{17} \mathrm{~m}^{-3}$ nuclei. This elevated number is much more than what was added by imposing shear to the neat iPP in the experiments of Housmans et al. (2009); mild flow raised the point-like nucleation density of the neat iPP by one to two decades before the shear effect saturated, i.e., adding typically $\sim 10^{13} \mathrm{~m}^{-3} \mathrm{nu}-$ clei. This indicates that, in the presence of nucleating agent, shear-induced point nucleation can be much more effective. This should be directly related to the nucleating agent, i.e., the local amplification of flow effects due to the presence of the nucleation particles, see for example Hwang et al. (2006), and not to a change in the rheological properties, which we expect to be very small due to the, in this respect, still very low space filling due to nuclei.

\section{Conclusions}

A new method to determine nucleation densities, based on rheometry, was used on quiescent and sheared sam-

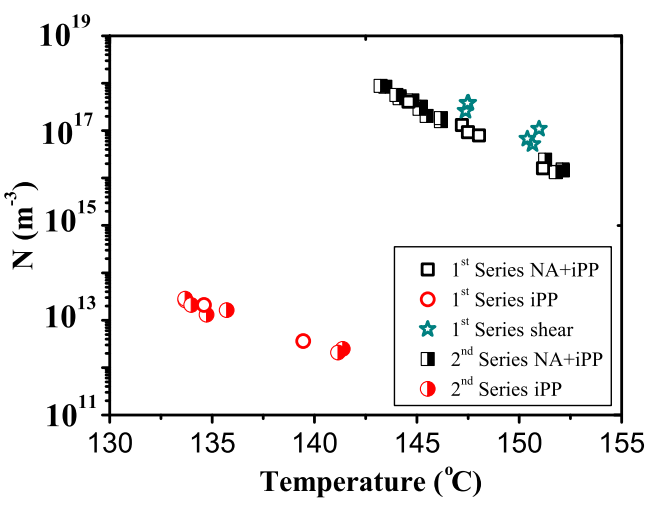

Fig. 10 Nucleation densities of iPP and NA-iPP under quiescent and shear conditions

ples of a neat and an artificially nucleated isotactic polypropylene. All calculated nucleation densities are summarized in Fig. 10. It is quantitatively shown that UPhthalocyanine is very effective for nucleating isotactic polypropylene. Moreover, it was found that the effect of shear is enhanced by the presence of the nucleating agent. This rheological method is easy to apply since it requires a standard rheometer, available in most academic and industrial labs. Finally, it was demonstrated that the temperature has to be carefully controlled in order to obtain quantitative number densities.

Acknowledgements This work is part of the Research Programme of the Dutch Polymer Institute (DPI), P.O. Box 902, 5600 AX Eindhoven, The Netherlands, project \#714.

Open Access This article is distributed under the terms of the Creative Commons Attribution Noncommercial License which permits any noncommercial use, distribution, and reproduction in any medium, provided the original author(s) and source are credited.

\section{References}

Avrami M (1939) Kinetics of phase change. I. General theory. J Chem Phys 7:1103-1112

Avrami M (1940) Kinetics of phase change. II. Transformationtime relations for random distribution of nuclei. J Chem Phys 8:212-224

Binsbergen FL, De Lange BGM (1970) Heterogeneous nucleation in the crystallization of polyolefins: part 2. Kinetics of crystallization of nucleated polypropylene. Polymer 11: 309-332

Boutahar K, Carrot C, Guillet J (1996) Polypropylene during crystallization from the melt as a model for the rheology of molten-filled polymers. J Appl Polym Sci 60:103-1996

Boutahar K, Carrot C, Guillet J (1998) Crystallization of polyolefins from rheological measurements-relation between the transformed fraction and the dynamic moduli. Macromolecules 31:1921-1929 
Byelov D, Panine P, Remerie K, Biemond E, Alfonso GC, De Jeu WH (2008) Crystallization under shear in isotactic polypropylene containing nucleators. Polymer 49:3076-3083

Christensen RM (1990) A critical evaluation for a class of micromechanics models. J Mech Phys Solids 38:379-404

Christensen RM, Lo KH (1979) Solutions for effective shear properties in three phase sphere and cylinder models. J Mech Phys Solids 27:315-330

Christensen RM, Lo KH (1986) Erratum: solutions for effective shear properties in three phase sphere and cylinder models. J Mech Phys Solids 34:639

Coppola S, Acierno S, Grizzuti N, Vlassopoulos D (2006) Viscoelastic behavior of semicrystalline thermoplastic polymers during the early stages of crystallization. Macromolecules 39:1507-1514

Eder G, Janeschitz-Kriegl H (1997) Structure development during processing: crystallization. In: Meijer HEH (ed) Processing of Polymers. Materials science and technology: a comprehensive treatment, no. 18. Wiley-VCH, Weinheim, pp 269-342

Evans UR (1945) The laws of expanding circles and spheres in relation to the lateral growth of surface films and the grainsize of metals. Trans Faraday Soc 41:365-374

Forstner R, Peters GWM, Meijer HEH (2009) A novel dilatometer for PVT measurements of polymers at high cooling - and shear rates. Int Polym Process 24:114-121

Hoffman JD, Davis GT, Lauritzen JI (1976) The rate of crystallization of linear polymer with chain folding. In: Hannay NB (ed) Treatise on solid state chemistry, vol 3. Plenum, New York, pp 497-614

Housmans JW, Steenbakkers RJA, Roozemond PC, Peters GWM, Meijer HEH (2009) Saturation of pointlike nuclei and the transition to oriented structures in flow-induced crystallization of isotactic polypropylene. Macromolecules 42:5728-5740

Hwang WR, Peters GWM, Hulsen MA, Meijer HEH (2006) Flow-induced crystallization of particle-filled polymers. Macromolecules 39(24):8389-8398

Khanna YP (1993) Rheological mechanism and overview of nucleated crystallization kinetics. Macromolecules 26: 3639-3643

Kolmogorov AN (1937) On the statistical theory of the crystallization of metals. Bull Acad Sci USSR, Math Series 3:355359 (in Russian)

Kristiansen M, Werner M, Tervoort T, Smith P, Blomenhofer M, Schmidt HW (2003) The binary system isotactic polypropylene/bis(3,4-dimethylbenzylidene)sorbitol: phase behavior, nucleation, and optical properties. Macromolecules 36:5150-5156
Kristiansen M, Tervoort T, Smith P, Goossens H (2005) Mechanical properties of sorbitol-clarified isotactic polypropylene: influence of additive concentration on polymer structure and yield behavior. Macromolecules 38:1046110465

Kurja J, Mehl NA (2001) Nucleating agents for semicrystalline polymers. In: Zweifel H (ed) Plastics additives handbook, 5th edn. Hanser, Munich, pp 949-972

Lamberti G (2004) A direct way to determine iPP density nucleation from DSC isothermal measurements. Polym Bull 52:443-449

Lee Wo D, Tanner RI (2010) The impact of blue organic and inorganic pigments on the crystallization and rheological properties of isotactic polypropylene. Rheol Acta 49:75-88

Menyhárd A, Gahleitner $\mathrm{M}$, Varga J, Bernreitner $\mathrm{K}$, Jääskeläinen P, Øysæd H, Pukánszky B (2009) The influence of nucleus density on optical properties in nucleated isotactic polypropylene. Eur Polym J 45:3138-3148

Pogodina NV, Winter HH, Srinivas S (1999) Strain effects on physical gelation of crystallizing isotactic polypropylene. J Pol Sci B Polymer Phys 37:3512-3519

Steenbakkers RJA (2009) Precursors and nuclei, the early stages of flow-induced crystallization. Ph.D. thesis, Technische Universiteit Eindhoven. Available at http://www.mate. tue.nl/mate

Steenbakkers RJA, Peters GWM (2008) Suspension-based rheological modeling of crystallizing polymer melts. Rheol Acta 47:643-665

Tanner RI (2002) A suspension model for low shear rate polymer solidification. J Non-Newton Fluid Mech 102:397-408

Tanner RI (2003) On the flow of crystallizing polymers I. Linear regime. J Non-Newton Fluid Mech 102:397-408

Van der Beek MHE, Peters GWM, Meijer HEH (2006) Classifying the combined influence of shear rate, temperature, and pressure on crystalline morphology and specific volume of isotactic (poly)propylene. Macromolecules 39:92789284

van Ruth NJL, Vega JF, Rastogi S, Martínez-Salazar J (2006) Viscoelastic behavior during the crystallisation of isotactic polypropylene. J Mater Sci 41:3899-3905

Wunderlich B (1973) Macromolecular physics, vol 2: crystal nucleation, growth, annealing. Academic, London

Zhu PW, Edward G (2004) Distribution of shish-kebab structure of isotactic polypropylene under shear in the presence of nucleating agent. Macromolecules 37:2658-2660

Zuidema H, Peters GWM, Meijer HEH (2001) Development and validation of a recoverable strain-based model for flowinduced crystallization of polymers. Macromol Theory Simul 10:447-460 